\title{
Molecular specific and cell selective cytotoxicity induced by a novel synthetic HLA-DR antibody mimic for lymphoma and leukemia
}

\author{
G.L. DeNARDO ${ }^{1}$, G.R. MIRICK ${ }^{1}$, S. HOK ${ }^{2}$, S.J. DeNARDO ${ }^{1}$, \\ L.A. BECKETT ${ }^{1}$, G.N. ADAMSON ${ }^{1}$ and R.L. BALHORN ${ }^{2}$ \\ ${ }^{1}$ University of California, Davis Medical Center, Sacramento, CA 95816; \\ ${ }^{2}$ Lawrence Livermore National Laboratories, Livermore, CA 94551, USA
}

Received October 6, 2008; Accepted November 7, 2008

DOI: 10.3892/ijo_00000176

\begin{abstract}
Like rituximab, monoclonal antibodies reactive with human leukocyte antigen have potent antilymphoma activity. However, size limits their vascular and tissue penetration. To mimic monoclonal antibody binding, nanomolecules have been synthesized, shown specific for the $\beta$ subunit of HLA-DR10, and selective for cells expressing this protein. Selective high affinity ligands (SHALs) containing the 3-(2([3-chloro-5-trifluoromethyl)-2-pyridinyl]oxy)-anilino)-3oxopropanionic acid $(\mathrm{Ct})$ ligand residualized and had antilymphoma activity against expressing cells. Herein, we show the extraordinary potency in mice with human lymphoma xenografts of a tridentate SHAL containing this ligand. After titrating antilymphoma activity in cell culture, a randomized preclinical study of a tridentate SHAL containing the $\mathrm{Ct}$ ligand was conducted in mice with established and aggressive human lymphoma xenografts. Mice having HLA-DR10 expressing Raji B- or Jurkat's T-lymphoma xenografts were randomly assigned to receive either treatment with SHAL at a dose of 100 ng i.p. weekly for 3 consecutive weeks, or to be untreated. Primary end-points were cure, overall response rates and survival. Toxicity was also evaluated in these mice, and a USFDA general safety study was conducted in healthy Balb/c mice. In Raji cell culture, the threshold and $\mathrm{IC}_{50}$ concentrations for cytotoxic activity were 0.7 and $2.5 \mathrm{nmol}(\mathrm{pm} / \mathrm{ml}$ media), respectively. When compared to treated Jurkat's xenografts or untreated xenografts, Raji xenografts treated with the SHAL showed an $85 \%$ reduction in hazard of death $(\mathrm{P}=0.014 ; 95 \%$ confidence interval $32-95 \%$ reduction). There was no evidence for toxicity even after i.p. doses 2000 times greater than the treatment dose associated with cure of a majority of the mice with Raji xenografts. When compared with control groups,
\end{abstract}

Correspondence to: Dr Gerald L. DeNardo, 1508 Alhambra Blvd., Room 3100, Sacramento, CA 95816, USA

E-mail: gldenardo@ucdavis.edu

Key words: lymphoma, leukemia, therapy, nanomolecules, antibody, high affinity ligands, HLA-DR signaling treatment selectively improved response rates and survival in mice with HLA-DR10 expressing human lymphoma xenografts at doses not associated with adverse events and readily achievable in patients.

\section{Introduction}

Cell surface proteins, such as CD20, CD22 and MHCII HLA-DR, have proven to be attractive targets for therapeutics for non-Hodgkin's lymphoma (NHL). One of these proteins, human leukocyte antigen (HLA) is upregulated on the surface of malignant B lymphocytes when compared to normal B lymphocytes. Moreover, intracellular HLA-DR protein is even more abundant (1). HLA-DR proteins serve as transmembrane and cytoplasmic signaling receptors and as peptide shuttles for the immune system (2-4). The monoclonal antibody (MAb), Lym-1, binds to a well-characterized epitope on the $\beta$ subunit of HLA-DR and reacts with lymphoma tissue from about 90 and $50 \%$ of patients with B-cell lymphoma and leukemia, respectively (5-7). Although binding is restricted to the cell surface (8), Lym-1 is highly active against malignant B cells in culture and in mice (9-11).

To mimic MAb binding to HLA-DR10, while decreasing size, a series of selective high affinity ligands (SHALs) $<5 \mathrm{kDa}$ in size have been synthesized to bind in the Lym-1 epitopic region of the $\beta$ subunit of HLA-DR protein based on in silico modeling and experimental studies. Bidentate versions of these novel nanomolecules showed many desired characteristics in vitro and in mice $(12,13)$ but had no antilymphoma activity (14). Strikingly, a tridentate SHAL containing the Ct ligand (3-(2-([3-chloro-5-trifluoromethyl)-2-pyridinyl] oxy)-anilino)3-oxopropanionic acid) residualized inside and was cytotoxic for B-lymphoma cells in culture (1). Here, we titrate the cellselective antilymphoma activity of this SHAL, demonstrate its remarkable efficacy, selectivity and safety in mice with human lymphoma xenografts and show electron microscopic evidence for SHAL-induced autophagy.

\section{Materials and methods}

Reagents and cell lines. Murine (Peregrine Pharmaceuticals, Tustin, CA) and chimeric (A. Epstein, Los Angeles, CA) 
Lym-1, that bind to the $\beta$ subunit of HLA-DR10 expressed on malignant B-cells (8), and HLA-DR10 protein isolated from Raji Burkitt's B-cells using a Lym-1 affinity column, as described previously (12) were used as references. An HLA-DR10 expressing human B lymphoma cell line, Raji (American Type Culture Collection, Manassas, VA), grown in RPMI-1640 with $10 \%$ fetal calf serum, supplemented with sodium pyruvate, non-essential amino acids and antibiotics (Gibco/Invitrogen, Carlsbad, CA), at $37^{\circ} \mathrm{C}$ in a humidified, $5 \%$ $\mathrm{CO}_{2}$ atmosphere and a non-expressing human $\mathrm{T}$ lymphoma cell line, Jurkat's (American Type Culture Collection, Manassas, VA), grown as recommended by ATCC, were used for these experiments.

Drug design and chemistry. Using homology modeling, HLA-DR amino acid residues critical for Lym-1 binding were mapped on a 3-D model of the HLA-DR10 ß subunit (12). Cavities within the Lym-1 epitopic surface of the protein were identified using SPHGEN $(15,16)$. After computational ligand docking for each of the cavities, a combination of NMR spectroscopy, surface plasmon resonance (BIAcore 3000; Biacore, Piscataway, NJ) and competitive binding experiments were used to confirm ligand binding to HLA-DR10 protein. Sets of ligands were then linked to create a SHAL, as described previously $(1,12)$. Each SHAL was synthesized on chlorotritylchloride resin in a polyethylene column (Pierce Biotechnology, Inc.) using Fmoc solid phase chemistry to conjugate PEG monomer units and selected ligands through the $\alpha$ and $\varepsilon$ amines of the N-terminal lysine. To synthesize the tridentate SHAL, a dabsylvaline (Dv) ligand was attached to the terminal amine of a PEG spacer conjugated to the alpha amine of the 2nd lysine residue and a 4-[4-(4-chlorobenzyl)piperazino]-3nitrobenzenecarboxylic acid $(\mathrm{Cb})$ ligand was attached to the terminal amine of a PEG conjugated to the alpha amine of the 3rd lysine. Lastly, the Ct ligand was also attached to the $\varepsilon$ amine of the 3rd lysine (Fig. 1). Conversion to DOTA (or biotinylated) SHAL derivatives was accomplished, as described previously (1). The reaction was monitored by analytical high performance liquid chromatography (HPLC), and the DOTA (or biotinylated) derivatives were purified using reverse phase, high performance liquid chromatography (RP-HPLC). Analytic electrospray ionization-mass spectrometry (Agilent 1100 instrument, Waters Symmetry C18 column) was used to confirm the elemental and mass composition of the SHAL (molecular weight within $0.07 \%$ of the theoretical molecular weight). To examine SHAL binding to isolated or recombinant HLA-DR10 protein, surface plasmon resonance experiments were conducted, as previously described (12). SHAL binding was better than nanomolar and was blocked by Lym-1 addition.

Cytotoxicity assay. Raji cells harvested in log growth phase were centrifuged, resuspended in fresh media and counted in $10 \%$ trypan-blue dye to determine initial and subsequent viability, as previously described $(1,14)$. When untreated, cells continued to multiply and nonviable cells, initially $<5 \%$ of total cells, remained so over the course of the assays. To titrate cytotoxic activity, biotinylated tridentate SHAL at concentrations ranging between 0 and $7 \mathrm{nM}(\mathrm{pm} / \mathrm{ml}$ media) was incubated with $0.5 \times 10^{6}$ cells $/ \mathrm{ml}$ at $37^{\circ} \mathrm{C}$ in a $5 \% \mathrm{CO}_{2}$, humidified atmosphere (NAPCO, Portland, OR). After 1, 2 and
3 days, cells were resuspended in trypan blue dye and counted in a hemocytometer. The fractional viability and absolute number of viable and non-viable cells $/ \mathrm{ml}$ were determined.

Efficacy and toxicity in mice. Seven- to nine-week old female, athymic Balb/c nu/nu mice (Harlan Sprague Dawley, Inc., Frederick, MD) were maintained according to University of California animal care guidelines under pathogen-free conditions and on a normal ad libitum diet. Raji or Jurkat's cells harvested in log phase and having $>95 \%$ viability were implanted $\left(6 \times 10^{6}\right.$ cells) subcutaneously in the lower abdomen 3-4 days after the mice were irradiated (400 cGy) to suppress xenograft rejection. When xenografts reached $20-500 \mathrm{~mm}^{3}$ by caliper measurement, the mice were sorted by xenograft volume into 4 groups: SHAL-treated mice with Raji xenografts, SHAL-treated mice with Jurkat's xenografts, untreated mice with Raji xenografts or untreated mice with Jurkat's xenografts. Each mouse was injected with $100 \mu 1$ of PBS or 100 ng DOTA-chelated tridentate SHAL i.p. on days 0,7 and 14. Xenograft volume and survival were monitored for $\geq 84$ days. The mice were also weighed 2-3 times each week for 4 weeks then weekly. Blood counts were measured prior to each SHAL or PBS dose and weekly for 4 weeks after the final dose using a Z Series Coulter Counter (Beckman Coulter Inc., Hialeah, FL) and a phase contrast microscope (Zeiss, Jena, Germany).

Xenograft volumes were calculated by the formula for hemiellipsoids (17). Responses were characterized as a cure, xenograft completely disappeared and did not regrow by 84 days, the formal censor day (>210 days to the present) complete remission (CR), xenograft disappeared for at least 7 days, but regrew, and partial remission (PR), xenograft volume decreased by at least $75 \%$ and remained stable for at least 7 days. All cures at 84 days in SHAL-treated mice persisted over a 210-day observation time for the mice. If a xenograft became $>2000 \mathrm{~mm}^{3}$, the mouse was euthanized in accordance with University of California animal care guidelines and scored as a disease-related death.

Safety in mice. Each of 3 groups of 7 healthy Balb/c mice were given PBS, 20 or $200 \mu \mathrm{g}$ of DOTA-chelated SHAL i.p., that is, 200 or 2,000 times the SHAL efficacy dose. Immediately prior to intervention, the mice were observed, weighed and blood counts (red, white and platelets) obtained, then the mice were observed daily, weighed 3 times each week and blood counts measured weekly for 4 weeks after intervention.

Electron microscopy (EM). Raji xenografts $\left(53-132 \mathrm{~mm}^{3}\right)$ were harvested for EM from mice, untreated (control) and 2, 4 or $24 \mathrm{~h}$ after $100 \mathrm{ng}$ of biotinylated SHAL. Xenografts were fixed, as described (18), using $4 \%$ paraformaldehyde in 0.1 M Sorenson's phosphate buffer, $\mathrm{pH}$ 7.35. After treating with $4 \%$ uranyl acetate in $70 \%$ ethanol for one hour and LR White acrylic resin overnight, polymerization was achieved in a microwave (Pelco 34700 BioWave, Pella Inc., Redding, CA). Ultra-thin sections (Leica Ultracut UCT, Leica, Vienna, Austria) were captured on gold grids, floated on Strepavidin$20 \mathrm{~nm}$ gold (BB International from Ted Pella Inc.) rinsed and stained with uranyl acetate and lead citrate before viewing using a Philips CM120 Biotwin Lens (FEI, Hillsboro, OR, 

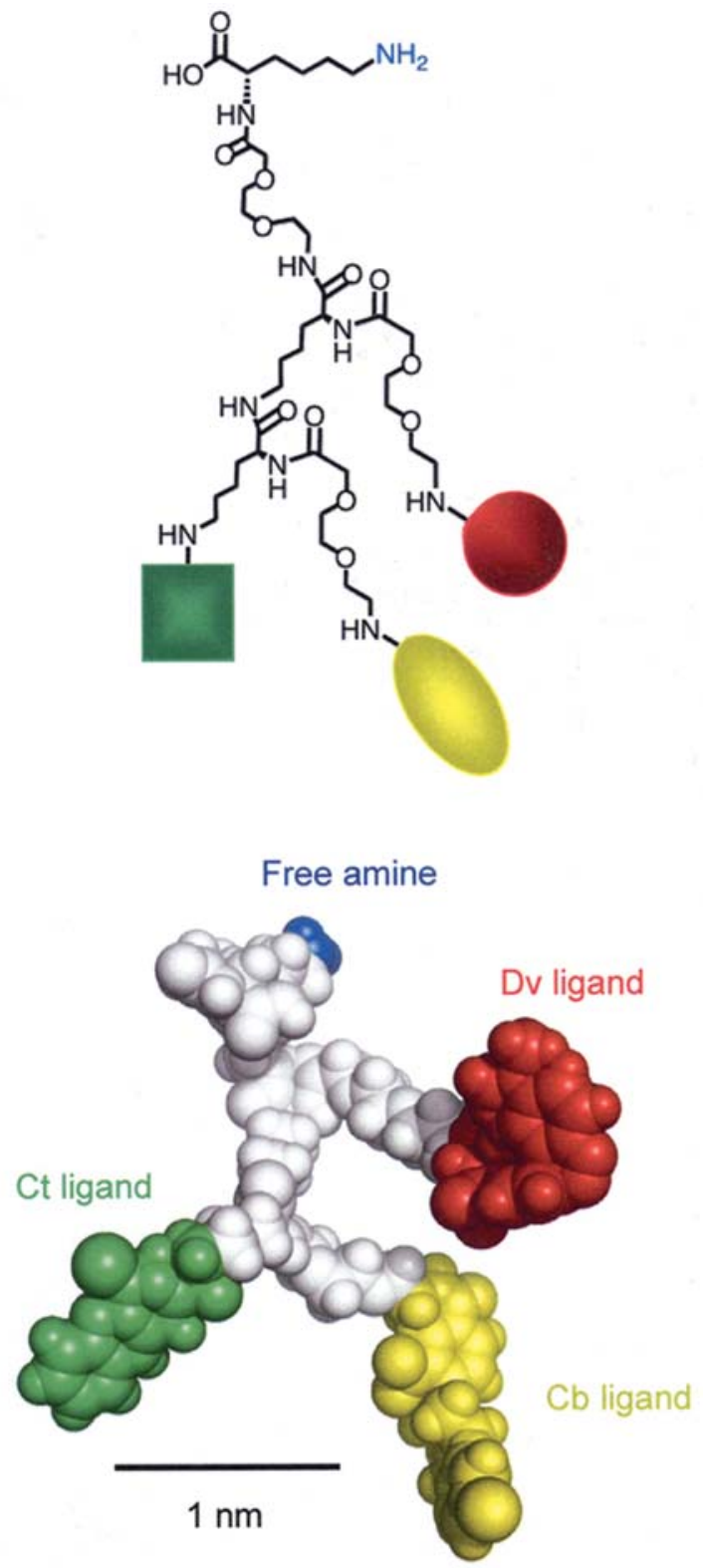

Figure 1. Structure of the tridentate SHAL containing the Ct ligand shown as a 2-D schematic (upper) and as a 3-D space-filling molecular structure (lower). The ligands used to produce this SHAL were dabsyl-L-valine (Dv), 4-[4-(4-chlorobenzyl)piperazino]-3-nitrobenzenecarboxylic acid (Cb) and (3-(2-([3-chloro-5-trifluoromethyl)-2-pyridinyl]oxy)-anilino)-3oxopropanionic acid) (Ct). The 1st lysine residue provided a free amine for covalent binding of moieties such as metal binding macrocycles and biotin. Additional lysine residues were used to create branch points in the linker and PEG monomers were used to provide the appropriate distance between the ligands. The three ligands were identified by docking to cavities in the B subunit of HLA-DR10, flanking the amino acid arginine 70 shown critical for Lym-1 binding and cytotoxicity. Functional molecules can be incorporated at specific positions along the linker by inserting additional lysine residues, and attaching them to the free epsilon amine of the primary lysine.

made in Eindhoven, The Netherlands and Gatan MegaScan, model 794/20, digital camera (2K X 2K), Pleasanton, CA. Gatan BioScan, model 792, Pleasanton, CA).

Biostatistical methods. Initial analysis compared response rates by exact tests and survival times by log-rank tests for untreated Raji and Jurkat's mice and found no significant differences, so these groups were pooled for subsequent



Figure 2. Titration of SHAL cytotoxic activity in Raji human HLA-DR10 expressing lymphoma cells. Absolute number of non-viable cells observed 1, 2 or 3 days after addition of SHAL at concentrations shown. SHAL threshold and $\mathrm{IC}_{50}$ concentrations were determined at 2 days to be 0.7 and $2.5 \mathrm{nM}(\mathrm{pm} / \mathrm{ml}$ media), respectively (mean $\pm \mathrm{SD})$.

analysis. Response rates for treated Raji, treated Jurkat's and untreated mice were compared by $\chi^{2}$ and exact tests. Survival times were censored at 84 days for mice who had not died or been euthanized because of tumor growth before that time. Times were summarized descriptively by Kaplan-Meier curves and median survival and 95\% confidence limits were obtained by the product-limit estimate. The two treated groups were compared to the untreated using a log-rank test for homogeneity across groups, followed by a proportional hazards model to estimate the effect of treatment compared to no treatment. Analyses were carried out using SAS/SAT ${ }^{\circledR}$ software (19). All tests were two-sided at level 0.05.

\section{Results}

Cytotoxicity assay. When untreated, non-viable (dead) Raji cells remained $<5 \%$ of the total cells over the 3 days of observation. Raji cells treated with SHAL [over a concentration range of $0-70 \mathrm{nM}(\mathrm{pm} / \mathrm{ml}$ media)] showed more dead cells, both fractionally and absolutely, over the 3-day period when compared to the untreated controls. The maximum number of non-viable cells was observed on day 2 at SHAL concentrations between 2.3 and $7 \mathrm{nM}$, and the threshold and $\mathrm{IC}_{50}$ SHAL cytotoxic concentrations were 0.7 and $2.5 \mathrm{nM}(\mathrm{pm} / \mathrm{ml}$ media), respectively (Fig. 2).

Efficacy and toxicity. Regression in treated Raji xenografts typically began $\sim 7$ and reached complete remission $\sim 30$ days after initial SHAL treatment (Fig. 3). Although censored at 84 days, all cures in SHAL-treated Raji xenografts have persisted to the present, seemingly representing permanent cures. Because there were no significant differences in response and survival of untreated Raji and Jurkat's mice $(\mathrm{P}>0.08)$ they were combined for comparison to treated mice. Mice with Raji xenografts and treated with SHAL had a $69 \%$ cure rate and $77 \%$ overall response rate, significantly better than treated mice with Jurkat's xenografts $(0 \%)$ and untreated mice $(15 \%$ response) $(\mathrm{P}<0.001)$ (Table I). The median survival 

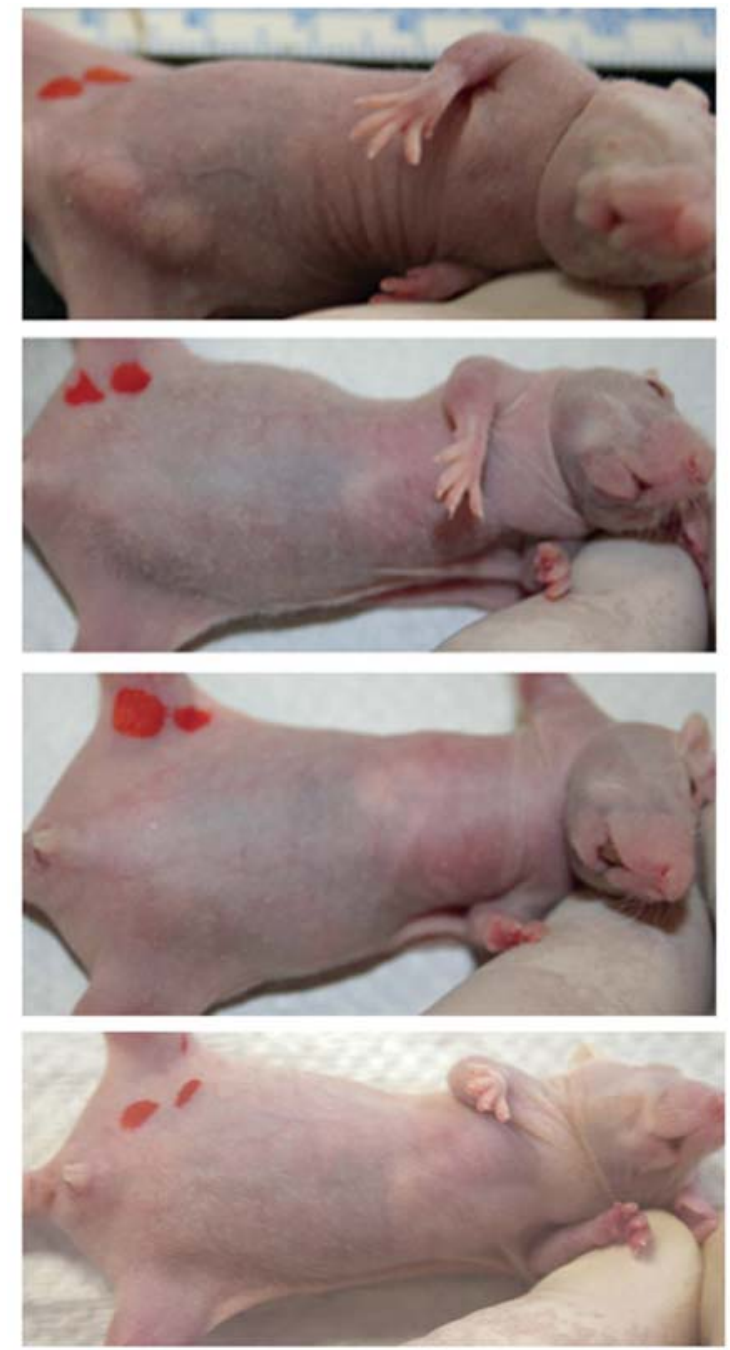

Figure 3. Photographic evidence for cure in a mouse with a Raji xenograft; before SHAL treatment (upper), one week after the initial and a second $100 \mathrm{ng}$ dose of SHAL i.p. By three weeks after the initial dose the xenograft had completely regressed and represented a cure that persisted over the period of observation of the mouse ( $>84$ days; lower).

Table I. Athymic mice with lymphoma xenografts, SHAL treated or untreated.

\begin{tabular}{lrcr}
\hline Treatment group & Mice & $\begin{array}{l}\text { Response (\%) } \\
\text { Cure (\%) }\end{array}$ & ORR (\%) \\
\hline Raji & 13 & 69 & 77 \\
Jurkat's & 9 & 0 & 0 \\
Untreated & 13 & 15 & 15 \\
\hline
\end{tabular}

${ }^{\mathrm{a} O v e r a l l ~ r e s p o n s e ~ r a t e . ~}$

times were 25 days for untreated mice and 21 days for treated mice with Jurkat's xenografts. The median survival time for treated mice with Raji xenografts was not estimable because almost all mice were alive at 84 days (Fig. 4). The proportional hazards model estimated that Raji mice had $\sim 85 \%$ reduction

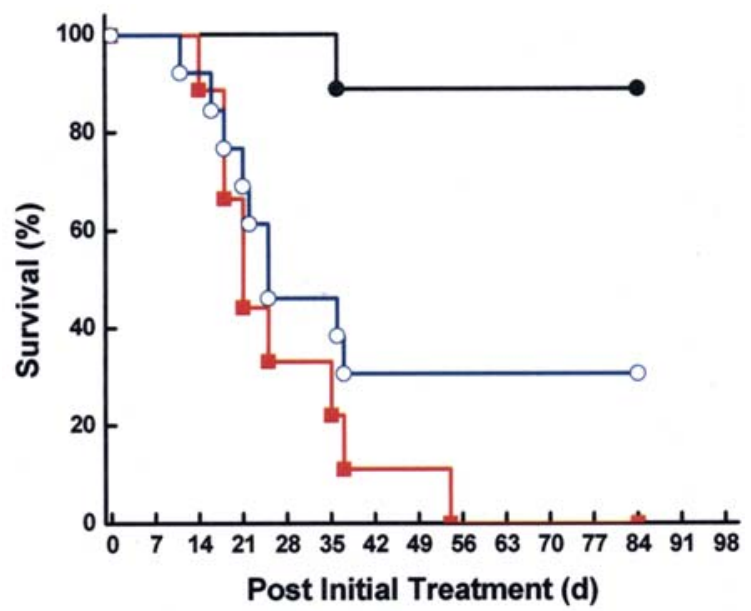

Figure 4. Overall survival effect of SHAL in mice with established Raji or Jurkat's lymphoma xenografts. Mice with Raji or Jurkat's xenografts were treated with $100 \mathrm{ng}$ SHAL i.p. once weekly for each of 3 consecutive weeks, or untreated. Mice with treated Raji xenografts (dark circle) had the longest survival while mice with treated Jurkat's xenografts (square) did not survive past 56 days. Two untreated mice (empty circle) with enlarging xenografts survived 84 days

in hazard of death compared to untreated and treated Jurkat's mice $(\mathrm{P}<0.001)$.

Safety in mice. All of the mice in the general safety study, including those given 2000 times the SHAL dose used in the efficacy trial, gained weight, showed stable blood counts and no adverse effects during 4 weeks of observation, thereby exceeding the requirements of the US FDA 'general safety' test guidelines.

Electron microscopy. Raji xenograft EM from untreated mice showed healthy cells having well-defined cellular and nuclear membranes, and cytoplasmic organelles, including reticular endothelium, Golgi, and liposomes (Fig. 5). In contrast, Raji EM, harvested after SHAL treatment, were characterized by highly condensed nuclear chromatin and fragmented organelles, findings consistent with autophagicy (programmed) cell death.

\section{Discussion}

Proteins on the surface of malignant cells have been used to identify them and to serve as targets for therapy and imaging. The class II major histocompatibility, human leukocyte antigens (HLA) are abundant both on the surface and inside malignant B-lymphocytes $(1,8)$. These proteins are major signaling receptors for cell death $(2,4)$. Whereas intact MAbs recognize malignant cells selectively, size limits their blood clearance and tissue penetration. Based on predictions from in silico modeling and from empiric testing, small organic ligands have been selected to bind to sites within the Lym-1 MAb epitopic region of HLA-DR10. By covalently linking sets of these ligands together, SHALs have been generated to target B-cell derived lymphomas and leukemias (20). These novel nanomolecules mimic the affinity and selectivity of MAbs because of three-dimensional interactions made between multiple ligands in the SHAL and multiple sites 

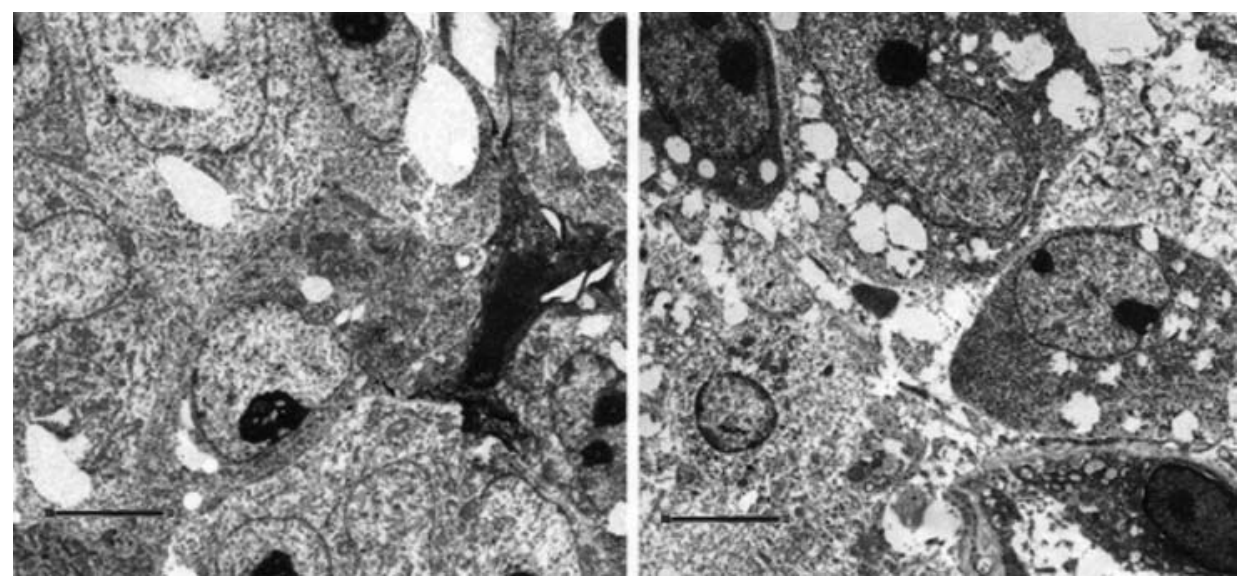

Figure 5. Electron micrographs of Raji xenografts excised from an untreated mouse and a mouse $24 \mathrm{~h}$ after SHAL treatment. Cellular structures were normal for this aggressive xenograft, untreated (left), whereas cells in the SHAL-treated xenograft showed condensed and fragmented chromatin and loss of cytoplasmic structures consistent with autophagic death (right) (bar, $5 \mu \mathrm{m}$ ).

located within the epitope of the target protein. SHALs are taken up rapidly by HLA-DR expressing xenografts and clear quickly from normal tissues (13) because the SHAL is 50 times smaller than an immunoglobulin $\mathrm{G}$ molecule. Histochemical analyses has shown that SHALs bind to NHL tissues from patients and from mice (12). Previous studies have shown that the SHALs readily enter cells, but they do not leave cells that express HLA-DR10 and related HLA-DRs, behaving like a lobster and its trap (1).

These SHALs cross cell barriers efficiently, seemingly unaffected in HLA-DR10 expressing cells by cellular pumping mechanisms. SHALs containing a $\mathrm{Ct}$ (3-(2-([3-chloro-5trifluoromethyl)-2-pyridinyl]oxy)-anilino)-3-oxopropanionic acid) ligand residualize inside and exhibit potent antilymphoma activity against live human HLA-DR10 expressing lymphoma cells (13). The present experiments demonstrate that the $\mathrm{Ct}$ ligand containing tridentate SHAL that has an $\mathrm{IC}_{50}$ of $2.5 \mathrm{nM}$ $(\mathrm{pm} / \mathrm{ml}$ media) for HLA-DR10 expressing cells in culture also exhibits remarkable efficacy in mice with Raji xenografts. Permanent cures were achieved in $69 \%$ of these mice at a SHAL treatment dose 2000-fold less than the maximum dose tested and confirmed to be safe in healthy mice. The antilymphoma effect was only observed in mice with xenografts expressing HLA-DR10; Jurkat's xenografts were not affected by SHAL treatment. Because mice treated with 2000 -fold more SHAL did not exhibit signs of adverse events, the SHAL seems to have an exceptional margin of safety as a potential therapeutic. It will be important in the future to conduct a dose response study with the tridentate SHAL in mice to ascertain whether or not the cure rate can be increased with a larger SHAL dose.

Electron micrographs of xenograft tissue from mice treated with the SHAL confirmed the SHAL's cytotoxicity to the Raji lymphoma cells. Extensive vacuolization and loss of cell structure suggest the possibility that SHAL binding to HLA-DR induced cell signaling that ultimately led to apoptosis and autophagic cell death. More extensive study will be required to confirm this hypothesis and identify the mechanism of action of the SHAL.

The results provide convincing evidence that SHALs have extraordinary potential as novel nanomolecules for targeting lymphoma and leukemia for molecular therapy. SHALs represent an attractive alternative to their biological counterparts, because these chemicals are $\sim 50$ times smaller, less expensive, easier to produce with consistency, stable and expected to have a long shelf-life and be effective when given orally. Furthermore, SHAL-based therapeutics can transport and residualize other agents near critical sites inside these malignant cells. The SHAL production platform is efficient, flexible, and permits rapid synthesis and modifications.

\section{Acknowledgements}

This study was supported by National Cancer Institute Grant PO1-CA47829 and at Lawrence Livermore National Laboratory under auspices of Department of Energy Contract DE-AC52-07NA27344.

\section{References}

1. DeNardo GL, Natarajan A, Hok S, Mirick G, DeNardo SJ, Corzett M, et al: Nanomolecular HLA-DR10 antibody mimics; a potent system for molecular targeted therapy and imaging. Bioconjug Chem (In press).

2. Leveille C, Cataigne JG, Charron D and Al-Daccak R: MHC class II isotype-specific signaling complex on human B cells. Eur J Immunol 32: 2282-2289, 2002.

3. Klemm JD, Schreiber SL and Crabtree GR: Dimerization as a regulatory mechanism in signal transduction. Annu Rev Immunol 16: 569-592, 1998.

4. Lane PJ, McConnell FM, Schieven GL, Clark EA and Ledbetter JA: The role of class II molecules in human B cell activation. J Immunol 144: 3684-3692, 1990.

5. DeNardo GL, DeNardo SJ, Goldstein DS, Kroger LA, Lamborn KR, Levy NB, et al: Maximum tolerated dose, toxicity, and efficacy of ${ }^{131}$ I-Lym-1 antibody for fractionated radioimmunotherapy of non-Hodgkin's lymphoma. J Clin Oncol 16: 3246-3256, 1998.

6. Rose LM, Gunasekera AH, DeNardo SJ, DeNardo GL and Meares CF: Lymphoma-selective antibody Lym-1 recognizes a discontinuous epitope on the light chain of HLA-DR10. Cancer Immunol Immunother 43: 26-30, 1996.

7. Rose LM, Deng CT, Scott S, Xiong CY, Lamborn KR, Gumerlock PH, et al: Critical Lym-1 binding residues on polymorphic HLA-DR molecules. Mol Immunol 36: 789-797, 1999.

8. Epstein AL, Marder RJ, Winter JN, Stathopoulos E, Chen FM, Parker JW, et al: Two new monoclonal antibodies, Lym-1 and Lym-2, reactive with human-B-lymphocytes and derived tumors, with immunodiagnostic and immunotherapeutic potential. Cancer Res 47: 830-840, 1987. 
9. DeNardo GL, Tobin E, Chan K, Bradt BM and DeNardo SJ: Direct anti-lymphoma effects on human lymphoma cells of monotherapy and combination therapy with CD20 and HLA-DR antibodies and yttrium-90 HLA-DR antibodies. Clin Cancer Res 11: 7075-7079, 2005.

10. Tobin E, DeNardo GL, Zhang N, Epstein AL, Liu C and DeNardo SJ: Combination immunotherapy with anti-CD20 and anti-HLA-DR monoclonal antibodies induces synergistic anti-lymphoma effects in human lymphoma cell lines. Leuk Lymphoma 48: 944-956, 2007.

11. Zhang N, Khawli LA, Hu P and Epstein AL: Lym-1-induced apoptosis of non-Hodgkin's lymphomas produces regression of transplanted tumors. Cancer Biother Radiopharm 22: 342-356, 2007.

12. Balhorn R, Hok S, Burke P, Lightstone FC, Cosman M, Zemla A, et al: Selective high affinity ligand antibody mimics for cancer diagnosis and therapy: initial application to lymphoma/ leukemia. Clin Cancer Res 13 (Suppl. 18): S5621-S5628, 2007.

13. DeNardo GL, Natarajan A, Hok S, Perkins J, Cosman M, DeNardo SJ, Lighstone FC, Mirick G, Miers LA, Yuan A, Xiong CY and Balhorn R: Pharmacokinetic characterization in xenografted mice of a series of first generation mimics for HLA-DR antibody, Lym-1, as carrier molecules to image and treat lymphoma. J Nucl Med 48: 1338-1347, 2007.
14. West J, Perkins J, Hok S, Balhorn R, Lightstone FC, Cosman M, et al: Direct antilymphoma activity of novel, first-generation 'antibody mimics' that bind HLA-DR10-positive non-Hodgkin's lymphoma cells. Cancer Biother Radiopharm 21: 645-654, 2006.

15. Kuntz ID, Blaney JM, Oatley SJ, Langridge R and Ferrin TE: A geometric approach to macromolecule-ligand interactions. J Mol Biol 161: 269-288, 1982.

16. Desjarlais RL, Sheridan RP, Seibel GL, Dixon JS, Kuntz ID and Venkataraqhavan R: Using shape complementarity as an initial screen in designing ligands for a receptor binding site of known three-dimensional structure. J Med Chem 31: 722-729, 1988

17. DeNardo GL, Kukis DL, Shen S, Mausner LF, Meares CF, Srivastava SC, et al: Efficacy and toxicity of 67Cu-2IT-BATLym-1 radioimmunoconjugate in mice implanted with Burkitt's lymphoma (Raji). Clin Cancer Res 3: 71-79, 1997.

18. Tait S, Gunn-Moore F, Collison JM, Huang J, Luberski C, Pedraza L, et al: An oligodendrocyte cell adhesion molecule at the site of assembly of the paranodal axo-glial junction. J Cell Biol 150: 657-666, 2000.

19. SAS Institue, Inc. SAS/STAT Version 9.0. 2004. Cary, NC: SAS Institute.

20. Hok S, Natarajan A, Balhorn R, DeNardo SJ, DeNardo GL and Perkins J: Synthesis and radiolabeling of selective high-affinity ligands designed to target non-Hodgkin's lymphoma and leukemia. Bioconjug Chem 18: 912-921, 2007. 